\title{
Molecular confinement of solid and gaseous phases of self-standing bulk nanoporous polymers inducing enhanced and unexpected physical properties
}

\author{
Javier Pinto $^{\text {a, }}$, Belen Notario ${ }^{\text {b }}$, Raquel Verdejo ${ }^{c}$, Michel Dumon ${ }^{d}$, Stephane Costeux ${ }^{\text {e, }}$ \\ Miguel Angel Rodriguez-Perez ${ }^{\mathrm{b}, * *}$ \\ ${ }^{a}$ Nanophysics - Smart Materials Group, Istituto Italiano di Tecnologia (IIT), Via Morego, 30, 16163 Genova, Italy \\ b Cellular Materials Laboratory (CellMat), Condensed Matter Physics Department, Paseo Belén N7, University of Valladolid, 47011 Valladolid, Spain \\ ${ }^{\mathrm{c}}$ Institute of Polymer Science and Technology (ICTP-CSIC), Juan de la Cierva, 3, Madrid 28006, Spain \\ d Laboratoire de Chimie des Polymères Organiques (LCPO), Institut National Polytechnique de Bordeaux (INP), Université de Bordeaux, 16 Avenue Pey \\ Berland, 33607 Pessac-Cedex, France \\ e The Dow Chemical Company, Dow Building Solutions, 1501 Larkin Center Dr., Midland, MI 48674, USA
}

\section{A R T I C L E I N F O}

Article history:

Received 13 December 2016

Received in revised form

8 February 2017

Accepted 13 February 2017

Available online 15 February 2017

\section{Keywords:}

Molecular chain confinement

Dielectric properties

$\mathrm{CO}_{2}$ foaming

\begin{abstract}
A B S T R A C T
In this work it is provided the first evidence of the polymer chains confinement within self-standing pore walls of nanoporous materials based on poly (methyl methacrylate) (PMMA). This was made possible by producing a series of porous samples with a wide range of pore sizes between $90 \mathrm{~nm}$ and $3 \mu \mathrm{m}$ using processes combining $\mathrm{CO}_{2}$ sorption, selective block copolymer swelling or homogeneous physical foaming. Mobility restrictions of the PMMA chains in the porous samples with pore size below $200 \mathrm{~nm}$ was consistently demonstrated with several experimental techniques, including differential scanning calorimetry, Raman spectroscopy, and broadband dielectric spectroscopy.

In addition, several scale-reduction phenomena related to the constitutive elements of the porous materials, both in the polymeric and gaseous phases, and to the porous architecture are identified. The significance of these phenomena on macroscopic electrical conductivity and permittivity of the nanoporous materials is demonstrated, and the presented observations support previous explanations of improved mechanical properties and thermal insulation of this type of nano-materials.
\end{abstract}

๑) 2017 Elsevier Ltd. All rights reserved.

\section{Introduction}

Nanoporous polymers are of great scientific and technological importance [1]. Their tunable chemistry, high surface area, and improved mechanical properties offer promising new applications in catalysis, filtration $[2,3]$, hydrogen storage $[4,5]$, gas separation and adsorption [6,7], energy conversion [8], and microelectronics $[9,10]$; while their superior thermal properties are suited for applications as high performance thermal insulators in building trade, aeronautics, or even space launch $[11,12]$. Several strategies have been developed for the production of these novel materials, often taking advantage of polymer blends or block copolymers self-

\footnotetext{
* Corresponding author.

** Corresponding author.

E-mail addresses: Sanz.Pinto@iit.it (J. Pinto), marrod@fmc.uva.es (M.A. Rodriguez-Perez).
}

assembly followed by swelling or removal of a dispersed nanometric phase [13-19]. In recent years, the use of physical blowing agents (e.g. $\mathrm{CO}_{2}$ ) to induce the nucleation of pores inside homogeneous polymers with high chemical affinity for the blowing agent has gained popularity as this provides a pathway to exploit the unique properties of nanostructured porous polymers in advanced materials for large scale applications [19-26].

The nanometric architecture, defined by the presence of nanopores with sizes below $100 \mathrm{~nm}$, provides polymeric materials with a high surface area, in which the confinement of a gaseous phase leads to a drastic reduction of thermal conductivity (i.e. the Knudsen effect already demonstrated in aerogels and porous ceramics) [27], as recently shown experimentally in bulk nanoporous materials (thick nanocellular foams) phase-separated poly (methyl methacrylate) (PMMA)-based systems [28]. Some indirect evidence of changes in the polymer phase behavior within these nanoporous materials were found, along with an enhancement of several 
mechanical properties compared to microcellular foams [29]. Such improvements were explained by a possible confinement of the polymer chains into pore walls with nanometric dimensions below $60 \mathrm{~nm}$, by analogy to thin films [30]. Yet no experimental evidence exists of a relation between a hypothetical polymer chains confinement in the nanometric polymer matrix and macroscopic physical properties of bulk nanoporous polymers (i.e. self-standing samples with significant thickness of about or over $1 \mathrm{~mm}$, or thousands of times thicker than the size of the pores within), in particular when materials have a closed porous structure.

\section{Experimental section}

Materials: Neat poly (methyl methacrylate) homopolymer (PMMA, $T_{g}=112^{\circ} \mathrm{C}, \rho=1.18 \mathrm{~g} / \mathrm{cm}^{3}, M_{w}^{P M M A} \approx 83000 \mathrm{~g} / \mathrm{mol}, M_{n}^{P M M A}$ $\approx 43000 \mathrm{~g} / \mathrm{mol}, I_{p}^{P M M A} \approx 1.9$ ) and a triblock copolymer poly (methyl methacrylate)-co-poly (butyl acrylate)-co-poly (methyl methacrylate) (MAM, $36 \mathrm{wt} \%$ poly (butyl acrylate), $\rho=1.08 \mathrm{~g} / \mathrm{cm}^{3}, M_{w}^{M A M} \approx$ $180000 \mathrm{~g} / \mathrm{mol}, M_{n}^{M A M} \approx 85000 \mathrm{~g} / \mathrm{mol}, I_{p}^{M A M} \approx 2.1$ ) were gently provided by Altuglas-Arkema Company (France) in the form of pellets.

Fabrication of PMMA-based nanoporous polymers: 90/10 PMMA/ MAM blends containing $10 \mathrm{wt} \%$ of MAM were produced as follows. Both materials, PMMA and MAM, were dried in vacuum $(680 \mathrm{~mm}$ $\mathrm{Hg}$ ), at $80{ }^{\circ} \mathrm{C}$ during $4 \mathrm{~h}$ before processing. Mixing and extrusion were carried out using a Scamex CE02 single-screw extruder (L/ $\mathrm{D}=28, \mathrm{~d}=45 \mathrm{~mm}$ ), with a temperature profile from 165 to $225^{\circ} \mathrm{C}$ at a screw speed of $60 \mathrm{rpm}$ in the desired proportions. Pellets were produced using a continuous cutting machine operating at the end of the line at a constant speed of $240 \mathrm{rpm}$. In a previous work it was demonstrated that PMMA/MAM blends produced by extrusion can present a self-assembled nanostructure that acts as a pattern in the production of the porous structure [3].

In a second step, neat PMMA and 90/10 PMMA/MAM were injected into bulk solid samples $\left(50 \times 15 \mathrm{~mm}^{2} \times 3 \mathrm{~mm}\right)$ using a small scale injection molding machine developed by DSM Xplore. The working temperature was fixed at $240{ }^{\circ} \mathrm{C}$, whereas mold temperature was set at $60{ }^{\circ} \mathrm{C}$. The injection pressure was fixed at $1 \mathrm{MPa}$. All samples were transparent and showed a good surface appearance as well as a good injection behavior, without air bubbles inside the parts (see Supplementary Information, Fig. S1).

Foaming experiments were carried out in a high pressure vessel provided by TOP Industry (France), with a capacity of $300 \mathrm{~cm}^{3}$ and capable of operating at maximum temperature of $250{ }^{\circ} \mathrm{C}$ and maximum pressure of $40 \mathrm{MPa}$. The reactor is equipped with an accurate pressure pump controller provided by Teledyne ISCO, and controlled automatically to keep the temperature and pressure at the desired values. The $\mathrm{CO}_{2}$ vessel temperature and pressure were monitored in the course of the process. Thus a collection of experiments was performed in a modified solid state foaming process. The usual solid state foaming process with amorphous polymers has three stages: the saturation (under fixed gas pressure and temperature), gas desorption during and after the pressure release (to room pressure and temperature), and foaming of the sample (at a temperature over or around the $T_{g}$ of the plasticized polymer). However, the actual glass transition temperature of PMMA- $\mathrm{CO}_{2}$ systems can reach values close to room temperature, even below room temperature in some particular conditions, so in this work the desorption (at room temperature) and foaming stages (also performed at room temperature) are not clearly separated.

In previous works it was stablished that the production of the porous structure in neat PMMA samples is obtained by a conventional gas nucleation and foaming process, whereas PMMA/MAM blends present a selective swelling of the soft phase (poly (butyl acrylate)) confined by the surrounding PMMA matrix [31].
In this study, bulk porous polymeric structures with pore sizes in the micrometric and nanometric range for neat PMMA, and only in the nanometric range for PMMA/MAM blends were obtained using $\mathrm{CO}_{2}$ as physical blowing agent (Table 1 ). The gas saturation process was carried out at room temperature and pressures between 10 and $30 \mathrm{MPa}$ during $24 \mathrm{~h}$ to assure the complete dissolution of $\mathrm{CO}_{2}$ in the polymer. After this saturation process, foaming was triggered by releasing the pressure inside the vessel at a pressure drop rate between 10 and $30 \mathrm{MPa} / \mathrm{min}$ and carried out at room temperature, obtaining bulk white porous samples (see Supplementary Information, Fig. S1).

Characterization of solid and nanoporous PMMA-based materials: density of solid and porous polymers was determined by waterdisplacement method, based on Archimedes' principle. In addition, both kind of materials were analyzed by differential scanning calorimetry (DSC, Mod. 862 Mettler), Raman spectroscopy (He-Ne Horiba JY Induram Laser (633 nm), Kaiser Raman OSI head MKII, and Kaiser spectrometer OSI HoloSpec), DC electrical resistivity (Keithley 6105 Resistivity Adapter), and broadband dielectric spectroscopy (BDS, Alpha high-resolution dielectric analyzer). Porous polymers were studied by scanning electron microscope (SEM, model Quanta 200FEG, FEI) to measure the pore size, pore density, and polymer wall thickness. More details about the characterization procedures can be found in the Supplementary Information.

Estimation of the confinement effect boundary and radius of gyration of PMMA: some studies about the confinement effect on the chain conformation relate the appearance of this effect with the characteristics of the polymer chains under study. In particular, Kraus et al. [32] determined, both experimentally and by modelling, that the confinement effect on thin films appears when the films thickness reaches a value below six times the radius of gyration of the polymer chains. As the pore walls can be considered two-dimensional structures similar to thin films, we have estimated the pore wall thickness values where confinement effect should be noticed, using that relationship between the radius of gyration and the confinement effect, and determining the radius of gyration with the equation proposed for PMMA by Kirste et al. [33] (Equation (1)).

$<r^{2}>=0.096 \cdot M_{w}^{0.98}$

Where $M_{w}$ is the average molecular weight of the PMMA chains. It is obtained a value of $7.97 \mathrm{~nm}$ for the radius of gyration of our PMMA, and therefore the expected thickness boundary for the confinement effect should be about $47 \mathrm{~nm}$.

Analysis of the Raman spectra and selection of relative intensities: the analysis of the Raman spectra was carried out by analyzing the evolution of four relative intensities between six peaks corresponding to vibrational modes of the pendant groups. The peaks selected in this study corresponds to $812,968,1452,1736$, and $2954 \mathrm{~cm}^{-1}$ (Fig. S2, see Supplementary Information). Relative intensities under study were selected according to the following criteria: for each pair of peaks the intensity of the peak of the pendant group or vibrational mode that needs less volume is divided by the intensity of the peak of the pendant group or vibrational mode expected that needs more volume in the vibration. Pendant groups or vibrational modes that need more volume will be more sensible to the confinement (as they are going to be constrained first and before the others needing less volume), and therefore it can be expected that the studied ratios will be sensitive to the chain conformational changes, increasing when the volume that the chain can occupy is reduced. The intensity ratios under study were $\mathrm{Y}_{812} / \mathrm{Y}_{968}, \mathrm{Y}_{812} / \mathrm{Y}_{1452}, \mathrm{Y}_{1736} / \mathrm{Y}_{1452}$, and $\mathrm{Y}_{2954} / \mathrm{Y}_{1452}$.

For instance, in the case of the relative intensity $\mathrm{Y}_{812} / \mathrm{Y}_{968}$, the 
Table 1

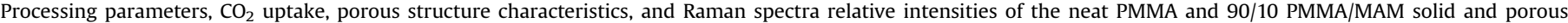
samples. Wave number of the Raman studied peaks are indicated as subscripts $\left(\mathrm{Y}_{\mathrm{nnnn}}\right.$, where $n n n n$ is the wavelength in $\left.\mathrm{cm}^{-1}\right)$.

\begin{tabular}{|c|c|c|c|c|c|c|}
\hline & Neat PMM & & & & & \\
\hline $\begin{array}{l}\text { Saturation Pressure } \\
\text { [MPa] }\end{array}$ & 0 (Solid) & 10 & 15 & 20 & 25 & 30 \\
\hline $\mathrm{CO}_{2}$ Uptake [wt.\%] & - & $24.3 \pm 0.2$ & $26.7 \pm 0.7$ & $28.1 \pm 0.7$ & $29.7 \pm 0.7$ & $31.5 \pm 0.1$ \\
\hline Relative Density & 1 & $0.66 \pm 0.01$ & $0.56 \pm 0.01$ & $0.53 \pm 0.01$ & $0.49 \pm 0.01$ & $0.46 \pm 0.01$ \\
\hline Average Pore Size [nm] & - & $3290 \pm 2037$ & $1460 \pm 977$ & $710 \pm 321$ & $200 \pm 120$ & $90 \pm 32$ \\
\hline $\begin{array}{l}\text { Average Pore Wall } \\
\text { Thickness [nm] }\end{array}$ & - & $308 \pm 112$ & $143 \pm 61$ & $105 \pm 42$ & $65 \pm 20$ & $30 \pm 9$ \\
\hline $\begin{array}{l}\text { Average Pore Density } \\
\left.\text { [pores } / \mathrm{cm}^{3}\right]\end{array}$ & - & $1.82 \times 10^{10} \pm 9.8 \times 10^{8}$ & $2.70 \times 10^{11} \pm 1.4 \times 10^{10}$ & $2.51 \times 10^{12} \pm 2.2 \times 10^{11}$ & $1.22 \times 10^{14} \pm 9.4 \times 10^{12}$ & $1.35 \times 10^{15} \pm 1.0 \times 10^{14}$ \\
\hline \multicolumn{7}{|c|}{ Raman Relative Intensities: } \\
\hline $\mathrm{Y}_{812} / \mathrm{Y}_{968}$ & $3.18 \pm 0.01$ & $2.44 \pm 0.01$ & $2.50 \pm 0.03$ & $2.43 \pm 0.01$ & $2.60 \pm 0.01$ & $2.85 \pm 0.02$ \\
\hline $\mathrm{Y}_{812} / \mathrm{Y}_{1452}$ & $1.97 \pm 0.01$ & $1.41 \pm 0.01$ & $1.45 \pm 0.01$ & $1.45 \pm 0.01$ & $1.61 \pm 0.03$ & $1.79 \pm 0.01$ \\
\hline $\mathrm{Y}_{1736} / \mathrm{Y}_{1452}$ & $0.53 \pm 0.01$ & $0.40 \pm 0.01$ & $0.42 \pm 0.01$ & $0.41 \pm 0.01$ & $0.42 \pm 0.01$ & $0.47 \pm 0.01$ \\
\hline \multirow[t]{2}{*}{$\mathrm{Y}_{2954} / \mathrm{Y}_{1452}$} & $8.50 \pm 0.02$ & $6.30 \pm 0.07$ & $6.47 \pm 0.01$ & $6.41 \pm 0.01$ & $6.55 \pm 0.12$ & $7.28 \pm 0.09$ \\
\hline & \multicolumn{6}{|c|}{ 90/10 РММА/МАМ } \\
\hline $\begin{array}{l}\text { Saturation Pressure } \\
\text { [MPa] }\end{array}$ & 0 (Solid) & 10 & 15 & 20 & 25 & \\
\hline $\mathrm{CO}_{2}$ Uptake [wt.\%] & - & $24.9 \pm 0.1$ & $27.5 \pm 0.1$ & $28.9 \pm 0.2$ & $30.6 \pm 0.2$ & \\
\hline Relative Density & 1 & $0.58 \pm 0.01$ & $0.54 \pm 0.01$ & $0.49 \pm 0.01$ & $0.43 \pm 0.01$ & \\
\hline Average Pore Size [nm] & - & $150 \pm 56$ & $160 \pm 58$ & $170 \pm 50$ & $190 \pm 69$ & \\
\hline $\begin{array}{l}\text { Average Pore Wall } \\
\text { Thickness [nm] }\end{array}$ & - & $21 \pm 10$ & $27 \pm 8$ & $33 \pm 15$ & $30 \pm 16$ & \\
\hline $\begin{array}{l}\text { Average Pore Density } \\
\left.\text { [pores } / \mathrm{cm}^{3}\right]\end{array}$ & - & $2.26 \times 10^{14} \pm 2.1 \times 10^{13}$ & $2.06 \times 10^{14} \pm 1.7 \times 10^{13}$ & $1.87 \times 10^{14} \pm 1.6 \times 10^{13}$ & $1.58 \times 10^{14} \pm 1.1 \times 10^{13}$ & \\
\hline \multicolumn{7}{|c|}{ Raman Relative Intensities: } \\
\hline $\mathrm{Y}_{812} / \mathrm{Y}_{968}$ & $3.09 \pm 0.02$ & $2.62 \pm 0.05$ & $2.66 \pm 0.04$ & $2.63 \pm 0.08$ & $2.66 \pm 0.08$ & \\
\hline $\mathrm{Y}_{812} / \mathrm{Y}_{1452}$ & $1.86 \pm 0.03$ & $1.57 \pm 0.05$ & $1.61 \pm 0.04$ & $1.61 \pm 0.03$ & $1.63 \pm 0.06$ & \\
\hline $\mathrm{Y}_{1736} / \mathrm{Y}_{1452}$ & $0.52 \pm 0.01$ & $0.44 \pm 0.01$ & $0.45 \pm 0.01$ & $0.44 \pm 0.01$ & $0.44 \pm 0.01$ & \\
\hline $\mathrm{Y}_{2954} / \mathrm{Y}_{1452}$ & $8.45 \pm 0.13$ & $6.40 \pm 0.10$ & $6.48 \pm 0.14$ & $6.43 \pm 0.07$ & $6.53 \pm 0.22$ & \\
\hline
\end{tabular}

$\mathrm{CH}_{3}$ group occupies more volume than the $\mathrm{C}-\mathrm{O}-\mathrm{C}$ group. Likewise, more volume is required to carry out the rock vibration of the $\mathrm{CH}_{3}$ group $\left(\mathrm{Y}_{968}\right)$ than to perform the stretching one of the $\mathrm{C}-\mathrm{O}-\mathrm{C}$ group $\left(\mathrm{Y}_{812}\right)$. Thus, in the nanometer scale, where movements are limited due to the reduction of pore walls thickness, the ratio between the intensity of the $\mathrm{C}-\mathrm{O}-\mathrm{C}$ group stretching and the $\mathrm{CH}_{3}$ group rock vibration should increase, as the $\mathrm{CH}_{3}$ rock vibration should be more sensitive to the confinement.

\section{Results and discussion}

Here, we obtain an experimental evidence of the confinement and scale-related effects on the physical properties of bulk nanoporous polymers. To provide such evidences we have produced a series of bulk porous materials with various degrees of confinement, which is achieved by inducing micro and nano porosity into homogeneous and nanostructured polymer matrices based on commercially available PMMA using $\mathrm{CO}_{2}$ as physical blowing agent. Moreover, the use of both fabrication strategies, i.e. swelling of a dispersed nanometric phase and the nucleation of pores in an homogeneous polymer, allow us to demonstrate that features in the nanometric architecture prevail in controlling the final properties over the procedure to develop the nanoporosity. Most specifically, the molecular confinement of the polymer chains in the nanometric pore walls was demonstrated by the progressive immobilization detected by Raman and Dielectric spectroscopy, which leads to a significant increment of the glass transition temperature of the nanoporous polymer matrix. In addition, below direct current it was found that the raising tortuosity of the porous architecture is compensated by an interfacial polarization phenomena (Maxwell Wagner Sillars), leading to bulk porous polymers with an electrical conductivity comparable to the bulk solids, and showing the potential to develop porous polymers with enhanced electrical conductivity with respect to the pristine solid polymer.

Scanning electron microscopy (SEM) (Fig. 1) shows the distinct porous architecture of neat PMMA and of nanostructured blends of $90 \mathrm{wt} \%$ PMMA and $10 \mathrm{wt} \%$ poly (methyl methacrylate)-co-poly (butyl acrylate)-co-poly (methyl methacrylate) (MAM). $\mathrm{CO}_{2}$ can induce porous structures in neat PMMA with increasing pore density and decreasing average pore sizes from $3 \mu \mathrm{m}$ to $90 \mathrm{~nm}$ by modifying the saturation pressure during the production process (Table 1). In contrast, the same procedure with the 90/10 PMMA/ MAM blend consistently leads to porous structures with pore sizes below $200 \mathrm{~nm}$, due to the controlled swelling of the poly (butyl acrylate) (PBA) dispersed domains [3,31], which results in rather constant pore densities that match the initial number density of dispersed domains. This controlled swelling of the PBA domains allows preventing the pore coalescence during the foaming process, in good agreement with the nano-voids formation mechanisms found in similar materials under mechanical deformation [34].

Details of the processing parameters and main characteristics of the porous structures obtained can be found in Table 1 and elsewhere [31]. The thickness of the pore walls $(\delta)$ decreases with the decrease of the pore size, reaching values between 20 and $65 \mathrm{~nm}$ for nanoporous samples with pore sizes below $200 \mathrm{~nm}$ (Table 1). To make progress toward understanding the confinement effects of nanoporous structures on amorphous thermoplastics polymer chains (e.g. PS and PMMA), differential scanning calorimetry (DSC) and Raman spectroscopy measurements were carried out. Previous studies demonstrated that chain confinement modified the glass transition temperature $\left(T_{g}\right)$ of the polymer, that can be either higher or lower than that of the bulk polymer [35,36], depending on the interfacial interactions between the polymer and the surrounding material (e.g. supporting substrate or nanoporous glass or alumina containers ...), surface mobility effects [37], and other 
a)

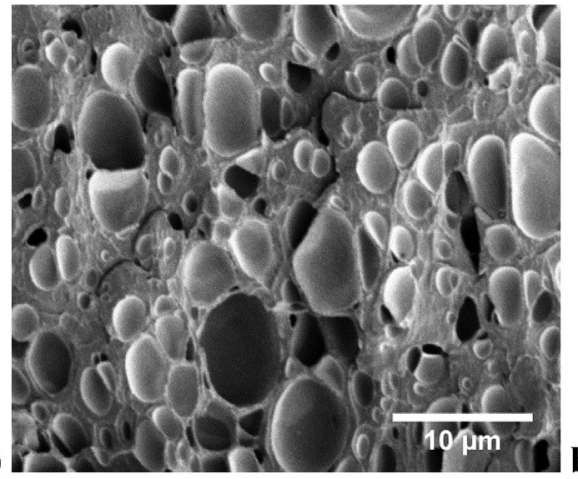

c)

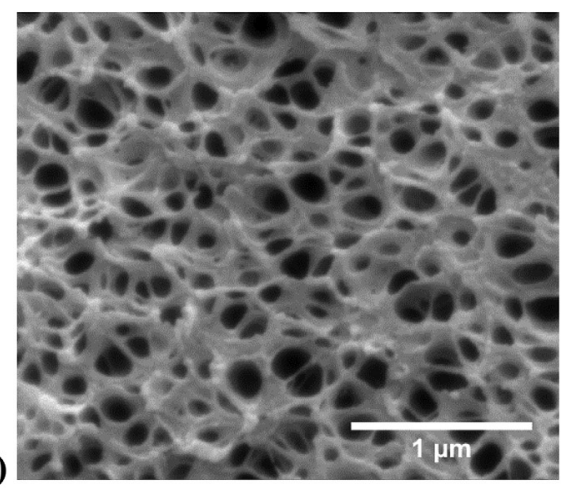

b)

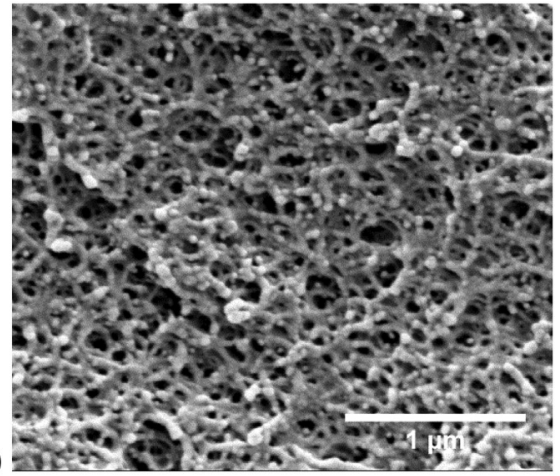

d)

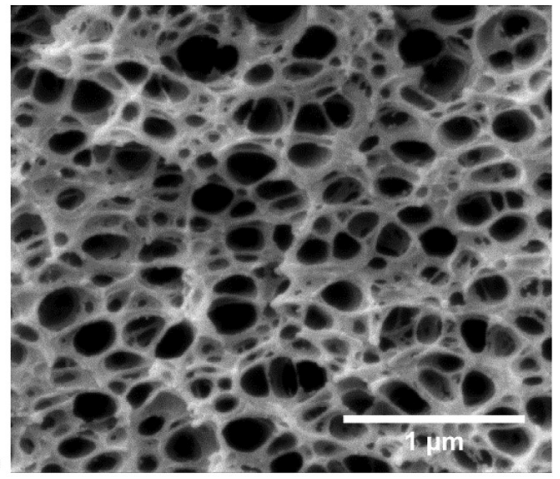

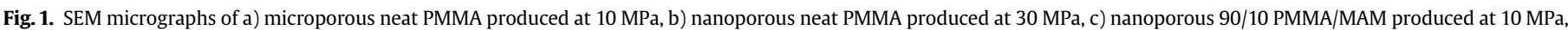
and d) nanoporous 90/10 PMMA/MAM produced at $25 \mathrm{MPa}$.

mechanisms yet to be identified [38]. Raman spectroscopy is a valuable technique to detect conformational changes of polymer chains induced by confinement [39]. The nanoporous walls are considered as self-standing confined materials (i.e. not supported by a substrate or in a container) and therefore, are free of interfacial constraints as opposed to model systems previously studied $[40,41]$ (see Supplementary Information).

Fig. 2a shows the $T_{g}$ increment between the bulk porous materials and solid polymer $\left(\Delta T_{g}=T_{g}^{\text {porous }}-T_{g}^{\text {solid }}\right)$ for various wall thicknesses. A gradual increase of $T_{g}$ is measured as the wall thickness decreases below $100 \mathrm{~nm}$ (i.e. nanoporous region), in contrast with the microporous region in which $\mathrm{T}_{\mathrm{g}}$ remains constant and equal to that of bulk dense PMMA. This effect is a direct consequence of PMMA chain confinement in thin pore walls. The thickness corresponding to the transition between the two regions is set around $100-150 \mathrm{~nm}$. A previous study has suggested the appearance of such confinement effect on thin films with thickness below six times the radius of gyration $\left(\left\langle r^{2}\right\rangle\right)$ of the polymer chains [32]. Here, the confinement region occurs at higher thicknesses than the suggested by Kraus et al. that corresponds to about $50 \mathrm{~nm}$ $\left(<r^{2}>_{P M M A}=7.97 \mathrm{~nm}\right.$, see Experimental Section $)$. It can be argued that such increase is due to the dynamic nature of the process used to make the materials, involving swelling by $\mathrm{CO}_{2}$ and biaxial stresses during pore expansion. However, although pore growing could induce some stretching on the polymer chains of thin pore walls, no prevailing effects of the biaxial stretching of PMMA chains on the $T_{g}$ of the pore walls can be expected. Previous works discarded significant effect of the stretching of non-crosslinked polymer films on the conformation of the polymer chains [42], and no relationship can be found between the hoop stress occurring at the surface of the pores and the $\mathrm{T}_{\mathrm{g}}$ evolution (see Supplementary Information). On the other hand, $\mathrm{CO}_{2}$ sorption can increase the radius of gyration of PMMA chains, thus inducing a local stretching of the molecules [43], thereby raising the thickness boundary at which the confinement is first observed. It should be noted that $\mathrm{CO}_{2}$ sorption effects alone cannot explain the increase in $T_{g}$, as several microporous PMMA samples made at high pressures experienced higher $\mathrm{CO}_{2}$ uptakes than nanoporous 90/10 PMMA/MAM made at lower pressure (Table 1), and yet do not show a $\mathrm{T}_{\mathrm{g}}$ increment. Therefore, our experiments demonstrate that the $T_{g}$ increase in nanoporous materials is directly related to polymer chains confinement (restriction of the molecular dynamics) in pore walls thinner than $100 \mathrm{~nm}$. Moreover, this behavior identified by DSC presents a good agreement with previous evidences about the evolution of the $T_{g}$ of this materials. In particular, studying the mechanical properties of this kind of materials it was found that the $\mathrm{T}_{\mathrm{g}}$, identified as the maximum of the $\alpha$-relaxation, increases in about $7^{\circ} \mathrm{C}$ when the pore size is decreased from 7-11 $\mu \mathrm{m}$ to 200-350 nm [29]; whereas the results obtained in this work shown a $T_{g}$ increase of about $6^{\circ} \mathrm{C}$ between microporous $(1-3 \mu \mathrm{m})$ and nanoporous ( $200 \mathrm{~nm}$ ) PMMA-based materials.

The analysis of the Raman relative intensities of the pendant groups provides further evidences of the molecular chain conformational modifications (Table 1, details of the studied peaks and example of Raman spectra can be found in the Supplementary Information). Extending the previous discussion, Raman analyses should account for both confinement effect inside the pore walls and the aforementioned influence of $\mathrm{CO}_{2}$ exposure on the polymer chains conformation. We note that nanoporous 90/10 PMMA/MAM with comparable pore wall thicknesses but $\mathrm{CO}_{2}$ uptakes ranging from 24.9 to $30.6 \mathrm{wt} \%$ present comparable values of the relative Raman intensities (Table 1). Thus, effects of $\mathrm{CO}_{2}$ uptake are negligible. Furthermore, the evolution of Raman relative peak intensities on neat PMMA porous samples indicates that ratios are rather constant for pore wall thicknesses above $100 \mathrm{~nm}$ (Table 1), but gradually increase by $10-20 \%$ as pore wall thicknesses fall below 


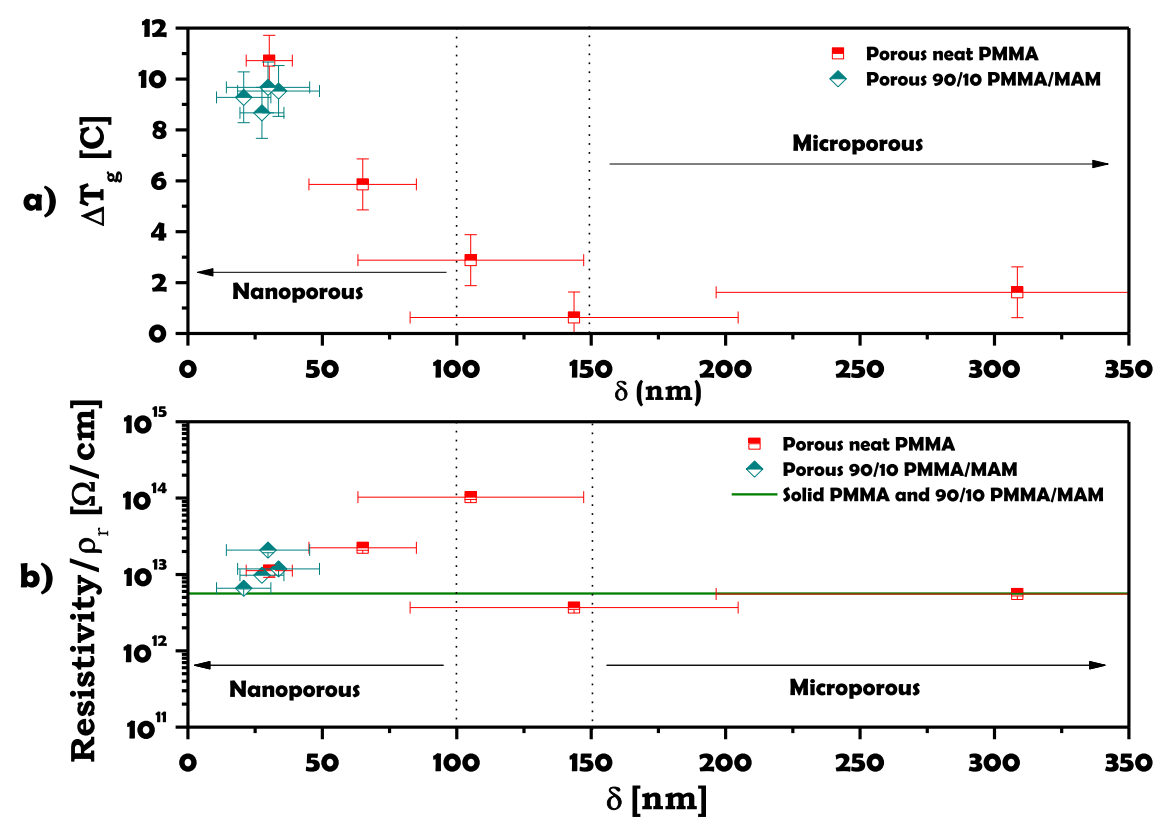

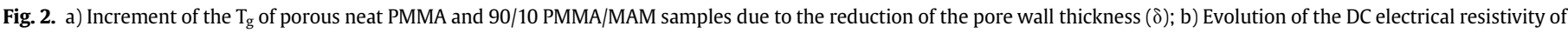

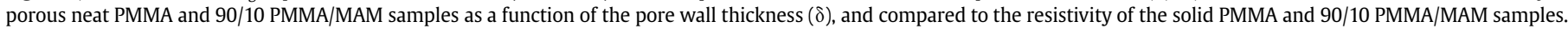

$100 \mathrm{~nm}$.

According to the selected relative intensities (see Experimental Section), such an increase is expected to relate to a vibrational mode hindrance of the larger pendant groups (e.g. here $\mathrm{CH}_{3}$ ). This is consistent with conformational changes due to polymer chains confinement in pore walls with thickness below about $100 \mathrm{~nm}$, in good agreement with the $T_{g}$ results.

Such conformational changes of polymer chains can provide an explanation for the mechanical properties enhancement found in bulk nanoporous PMMA materials [29], in the same way as the confinement of the gaseous phase provides the main explanation of the enhancement of the thermal insulation of these materials [28]. However, the reduction of the size of the constituent elements could modify the architecture of the porous material leading to modifications of their macroscopic properties. Such possibility was suggested as the result of the observed decrease of the solid phase contribution to the thermal conductivity, which was ascribed to an expected increase of the tortuosity of the porous architecture [28]. With the aim of validating this hypothesis, the DC electrical resistivity of the samples was measured, as an increase of the solid phase tortuosity should also increase the electrical resistivity of the porous material.

Fig. 2b shows the evolution of the DC electrical resistivity as a function of the pore wall thickness. An unexpected behavior is found: the resistivity of the microporous samples corresponds to the resistivity of the solid samples, but for pore wall thickness between 150 and $100 \mathrm{~nm}$ the resistivity rises about two orders of magnitude, and then decreases to the initial values of the solid and microporous samples. Comparison between Fig. $2 a$ and $2 b$ indicates that the evolution of the electrical conductivity is not directly related to the confinement effect of the solid PMMA phase. The sharp increment of the resistivity corresponds to a transition between micro and nanoporous materials (pore wall thickness from 150 to $100 \mathrm{~nm}$, corresponding to pore sizes from $1.5 \mu \mathrm{m}$ to $700 \mathrm{~nm}$ ). This increment of the DC electrical resistivity due to the reduction of the pore size (e.g. transition micro to nano porous materials) can be explained by the increase in tortuosity of the porous architecture, also observed in previous results on microporous materials
[44], and the reduction of the thermal conductivity in this kind of samples [28]. However, this hypothesis alone cannot explain the later progressive decrease in DC electrical resistivity when the pore size is decreased in the nanoporous domain. This points to an additional phenomenon that takes place in the nanoporous domain and overlaps with the increment of tortuosity.

Fig. 3a shows the dielectric constant $\left(\varepsilon^{\prime}\right)$ and the dielectric loss $(\varepsilon ")$ at room temperature of neat PMMA solid and porous samples determined by broadband dielectric spectroscopy over the frequency range from $10^{-2}-10^{5} \mathrm{~Hz}$. As expected, the dielectric constant decreases as the result of the increased free volume and the presence of entrapped air within the micro and nanopores. The solid and microporous samples present a dielectric relaxation, associated with local relaxation processes in amorphous polymers. This local relaxation is independent of the presence of the porous architecture and, hence, its relaxation time is the same in both the solid and the microporous materials. However, its intensity is drastically affected and decreases upon reduction of the pore size, disappearing for nanoporous materials. This intensity reduction and disappearance could be related to a progressive immobilization of the dipoles and polymer chains. Further evidence towards this argument is the decrease of the normalized dielectric strength as a function of the pore wall thickness (Fig. 3b). The dielectric strength $(\Delta \varepsilon)$ provides information on the contribution of the orientational polarization towards the dielectric permittivity, i.e. it provides information on the capacity of the dipoles to orient themselves, and is proportional to the density of dipoles involved in the relaxation process. Hence, the decrease of $\Delta \varepsilon$ with wall thickness is ascribed to a progressive reduction of the number of free dipoles able to rotate, due to confined configuration of the nanoporous system. A similar dependency of the $\Delta \varepsilon$ with films thicknesses was reported by Napolitano et al. for thin films [45].

Furthermore, nanoporous sample presents an abrupt increase of the loss permittivity at low frequencies, due to the presence of a conductivity component and an interfacial polarization phenomena, or Maxwell Wagner Sillars (MWS). MWS arises in heterogeneous materials, such as composites or blends, and is related to the accumulation of charges at the interfaces. MWS is very weak in all 
a)
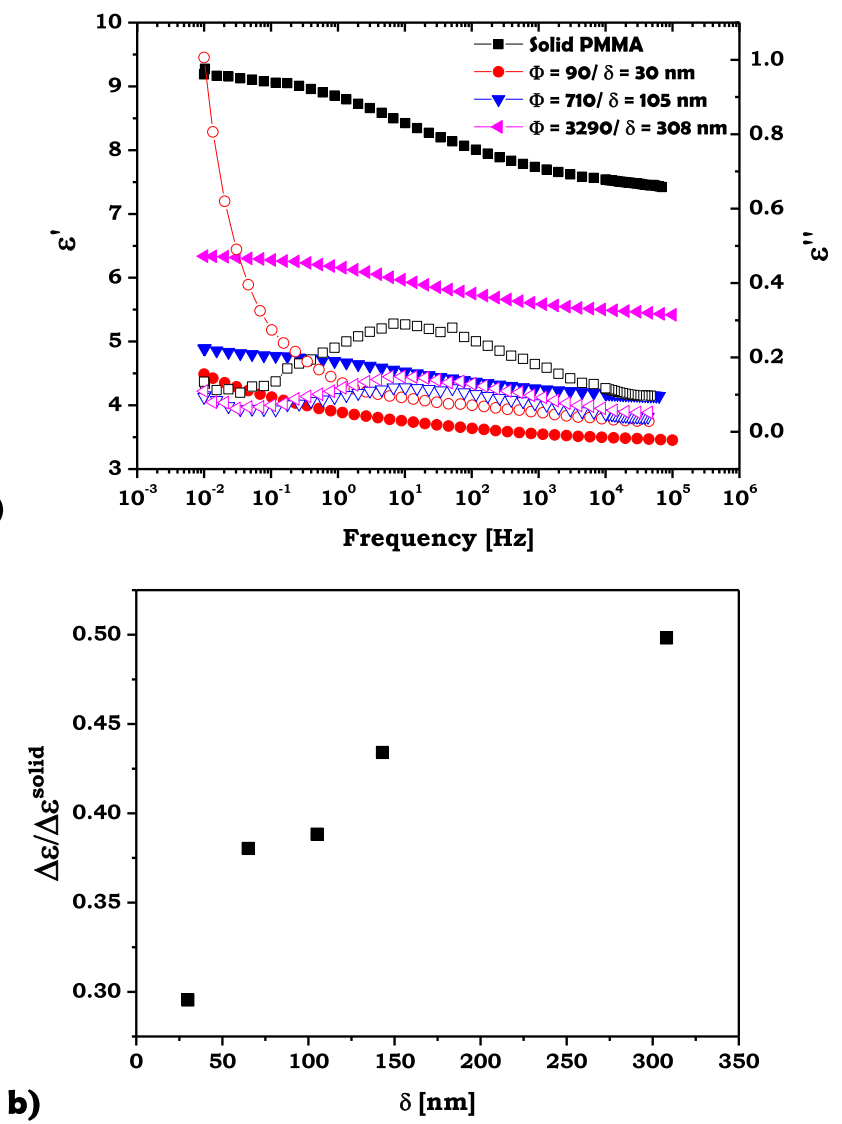

Fig. 3. a) Dielectric constant ( $\varepsilon^{\prime}$, solid symbols) and dielectric loss ( $\varepsilon^{\prime \prime}$, open symbols) as a function of frequency at room temperature $\left(23^{\circ} \mathrm{C}\right)$. b) Normalized dielectric strength $\left(\Delta \varepsilon / \Delta \varepsilon^{\text {solid }}\right)$ as a function of the pore wall thickness $(\delta)$.

microporous samples but it sharply rises in the nanoporous sample due to a combined effect of conductivity and MWS, visible as a change in the slope of $\varepsilon$ " at low frequencies. This conductivity could be the result of a possible capacitor-like behavior of opposing cell walls in nanopores presenting this accumulation of superficial charges. A classical explanation of this contribution of nanopores to the conductivity is the dielectric breakdown of the gas inside the capacitor-like nanopores due to the voltages employed in the characterization and the extremely small distances between the pore walls (between 90 and $200 \mathrm{~nm}$ in the samples that present this behavior). However, as previously demonstrated, the gas inside the nanopores presents a Knudsen diffusion regime due to the confinement [28], instead of classical Fickian diffusion. Therefore the assumption of a conventional dielectric breakdown could be inaccurate, making further studies necessary to identify the conductive mechanism through the capacitor-like nanopores.

Then, it can be expected that at high frequencies, where MWS does not take place, the sole mechanism should be the influence of the porous architecture (i.e. tortuosity), being responsible for the resistivity increment between micro and nanoporous samples. Accordingly, resistivity measurements at medium-high frequencies (Fig. S5, see Supplementary Information) show that microporous materials present a similar behavior as DC measurements (Fig. 2b), with resistivity values near to the solid PMMA results; on the contrary, nanoporous materials do not show the same behavior as DC measurements, increasing their resistivity when the pore size decreases, as can be expected assuming a rising tortuosity related to the decrease of the pore size. However, the picture changes substantially at low frequencies (Fig. 4) (resistivity measurements in the entire frequency range can be found in Fig. S5, see Supplementary Information). Resistivity differences between solid PMMA and porous samples with pore size about or over the micron remain practically constant; but the resistivity of nanoporous samples is decreased proportionally to the reduction of the frequency, reaching values near the resistivity of both solid and microporous PMMA. This effect can be related to the presence of the MWS phenomenon in nanoporous samples, explaining the behavior found in DC measurements where both effects, rising tortuosity and MWS, can take place in porous samples with pore sizes below the micron.

In summary, dielectric strength measurements demonstrate the polymer chains immobilization in the nanoporous materials. Moreover, the peculiar behavior of the electrical permittivity of PMMA-based nanoporous samples can be explained by the superposition of two phenomena: (1) a conductive mechanism through the confined gaseous phase of the nanopores detectable at low frequencies/DC, resulting in a reduction of the overall resistivity of the porous material proportional to the reduction of the pore size in the nanometer range; and (2) an increase in electrical resistivity of the solid phase due to the increase of the tortuosity when the pore size is decreased independently of current frequency (i.e. modification of porous architecture).

\section{Conclusions}

We demonstrate the presence of a confinement effect both in solid and gaseous phases of bulk nanoporous PMMA-based materials, produced by a $\mathrm{CO}_{2}$ sorption and selective swelling or foaming process. In the solid phase, this effect implies conformational changes and immobilization of the PMMA chains, which have been detected by Raman and Dielectric spectroscopy. As a consequence, the glass transition temperature of these materials presents an increase of up to $11^{\circ} \mathrm{C}$ in nanoporous samples with pore sizes about $90 \mathrm{~nm}$. As far as we know, this is the first demonstration of the confinement effect on self-standing three-dimensional porous polymeric samples with thickness of about $3 \mathrm{~mm}$ and porous structures with constitutive elements below $200 \mathrm{~nm}$, the results being independent of the pores generation mechanism (e.g. swelling of pre-existing self-assembled nanodomains or homogeneous pore nucleation in single phase PMMA). This confinement could be one of the key reasons explaining the improved mechanical properties of these materials previously observed. Moreover, we demonstrate the effect of a reduction of the scale of

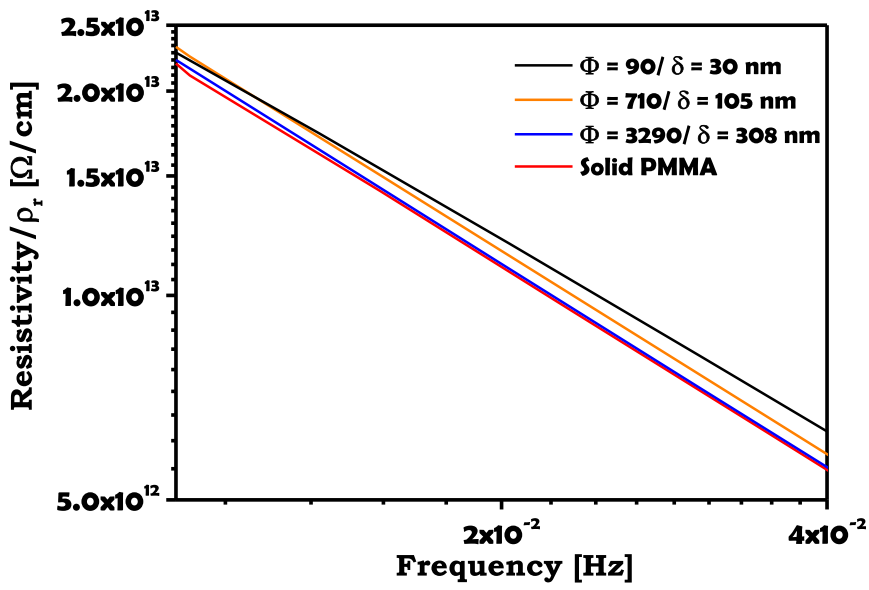

Fig. 4. Resistivity of most representative neat PMMA porous and solid samples measured in the low frequency range. 
constituent elements (from micro to nano) on the tortuosity of the porous architecture, which in turn has a significant impact on macroscopic physical properties as their electric and thermal conductivity. Finally, we observe an unexpected capacitor-like behavior in polymeric nanopores due to the appearance of a MWS phenomenon related to the confinement of the gaseous phase, with a significant influence on the electrical conductivity of these materials. This offers the potential for the development of unique sensors with very high surface area based on nanoporous polymeric materials and higher electrical conductivity than the pristine solid polymer.

\section{Acknowledgements}

Financial support from The Dow Chemical Company USA, FPI grant BES-2013-062852 (B. Notario) from the Spanish Ministry of Education, MINECO (MAT 2012-34901), and the Junta of Castile and Leon (VA035U13) is gratefully acknowledged. J. Pinto and B. Notario contributed equally to this work.

\section{Appendix A. Supplementary data}

Supplementary data related to this chapter can be found at http://dx.doi.org/10.1016/j.polymer.2017.02.046.

\section{References}

[1] B. Notario, J. Pinto, M.A. Rodriguez-Perez, Nanoporous polymeric materials: a new class of materials with enhanced properties, Prog. Mater. Sci. 78-79 (2016) 93-139.

[2] E.A. Jackson, Y. Lee, M.A. Hillmyer, ABAC tetrablock terpolymers for tough nanoporous filtration membranes, Macromolecules 46 (2013) 1484-1491.

[3] J. Pinto, M. Dumon, M.A. Rodriguez-Perez, R. Garcia, C. Dietz, Block copolymers self-assembly allows obtaining tunable micro or nanoporous membranes or depth filters based on PMMA; fabrication method and nanostructures, J. Phys. Chem. C 118 (2014) 4656-4663.

[4] J. Germain, J.M. Frechet, F. Svec, Nanoporous polymers for hydrogen storage, Small 5 (2009) 1098-1111.

[5] Z. Wang, S. Yuan, A. Mason, B. Reprogle, D.-J. Liu, L. Yu, Nanoporous porphyrin polymers for gas storage and separation, Macromolecules 45 (2012) 7413-7419.

[6] R.L. Martin, M.N. Shahrak, J.A. Swisher, C.M. Simon, J.P. Sculley, H.-C. Zhou, B. Smit, M. Haranczyk, Modeling methane adsorption in interpenetrating porous polymer networks, J. Phys. Chem. C 117 (2013) 20037-20042.

[7] A.M. Brockway, J. Schrier, Noble gas separation using $\operatorname{PG}-\operatorname{ESX}(X=1,2,3)$ nanoporous two-dimensional polymers, J. Phys, Chem. C 117 (2013) 393-402.

[8] A. Bentien, T. Okada, S. Kjelstrup, Evaluation of nanoporous polymer membranes for electrokinetic energy conversion in power applications, J. Phys Chem. C 117 (2013) 1582-1588.

[9] J.-W. Zha, H.-J. Jia, H.-Y. Wang, Z.-M. Dang, Tailored ultralow dielectric permittivity in high-performance fluorinated polyimide films by adjusting nanoporous characterisitics, J. Phys. Chem. C 116 (2012) 23676-23681.

[10] B. Notario, J. Pinto, R. Verdejo, M.A. Rodríguez-Pérez, Dielectric behavior of porous PMMA: from the micrometer to the nanometer scale, Polymer 107 (2016) 302-305.

[11] J.E. Fesmire, Aerogel insulation systems for space launch applications, Cryogenics 46 (2006) 111-117.

[12] C. Forest, P. Chaumont, P. Cassagnau, B. Swoboda, P. Sonntag, Polymer nanofoams for insulating applications prepared from CO2 foaming, Prog. Polym. Sci. 41 (2015) 122-145.

[13] Y. Wang, F. Li, An emerging pore-making strategy: confined swelling-induced pore generation in block copolymer materials, Adv. Mater. 23 (2011) 2134-2148.

[14] M. Lazzari, M.A. Lopez-Quintela, Block copolymers as a tool for nanomaterial fabrication, Adv. Mater. 15 (2003) 1583-1594.

[15] H.-P. Hentze, M. Antonietti, Porous polymers and resins for biotechnological and biomedical applications, Rev. Mol. Biotechnol. 90 (2002) 27-53.

[16] R. Zhang, H. Yokoyama, Fabrication of nanoporous structures in block copolymer using selective solvent assisted with compressed carbon dioxide, Macromolecules 42 (2009) 3559-3564.

[17] Z. Wang, Y. Wang, Highly Permeable and Robust Responsive Nanoporous membranes by selective swelling of triblock terpolymers with a rubbery block, Macromolecules 49 (2016) 182-191.
[18] L. Oss-Ronen, J. Schmidt, V. Abetz, A. Radulescu, Y. Cohen, Y. Talmon, Characterization of block copolymer self-assembly: from solution to nanoporous membranes, Macromolecules 45 (2012) 9631-9642.

[19] J. Pinto, M. Dumon, M.A. Rodriguez-Perez, Nanoporous polymer foams from nanostructured polymer blends, 2017, pp. 237-288.

[20] H. Yokoyama, L. Li, T. Nemoto, Tunable nanocellular polymeric monoliths using fluorinated block copolymer templates and supercritical carbon dioxide, Adv. Mater. 16 (2004) 1542-1546.

[21] H. Yokoyama, K. Sugiyama, Nanocellular structures in block copolymers with CO2-philic blocks using $\mathrm{CO} 2$ as a blowing agent: crossover from micro- to nanocellular structures with depressurization temperature, Macromolecules 38 (2005) 10516-10522.

[22] S. Costeux, CO2-Blown nanocellular foams, J. Appl. Polym. Sci. (2014) 131.

[23] L. Li, H. Yokoyama, T. Nemoto, K. Sugiyama, Facile fabrication of nanocellular block copolymer thin films using supercritical carbon dioxide, Adv. Mater. 16 (2004) 1226-1229.

[24] B. Krause, G.-H. Koops, 2N. F. A. v. d Vegt, M. Wessling, M. Wubbenhorst, J.V. Turnhout, Ultralow-k dielectrics made by supercritical foaming of thin films, Adv. Mater. 14 (2002) 1041-1046.

[25] B. Krause, K. Diekmann, N. F. A. v. d Vegt, M. Wessling, Open nanoporous morphologies from polymeric blends by carbon dioxide foaming, Macromolecules 35 (2002) 1738-1745.

[26] L. Li, T. Nemoto, K. Sugiyama, H. Yokoyama, CO2 foaming in thin films of block copolymer containing fluorinated blocks, Macromolecules 39 (2006) 4746-4755.

[27] L.W. Hrubesh, R.W. Pekala, Thermal properties of organic and inorganic aerogels, J. Mater Res. 9 (1994) 731-738.

[28] B. Notario, J. Pinto, E. Solorzano, J.A. de Saja, M. Dumon, M.A. Rodríguez-Pérez, Experimental validation of the Knudsen effect in nanocellular polymeric foams, Polymer 56 (2015) 57-67.

[29] B. Notario, J. Pinto, M.A. Rodríguez-Pérez, Towards a new generation of polymeric foams: PMMA nanocellular foams with enhanced physical properties, Polymer 63 (2015) 116-126.

[30] S. Kim, M.K. Mundra, C.B. Roth, J.M. Torkelson, Suppression of the tgnanoconfinement effect in thin poly(vinyl acetate) films by sorbed water, Macromolecules 43 (2010) 5158-5161.

[31] J. Pinto, M. Dumon, M. Pedros, J. Reglero, M.A. Rodriguez-Perez, Nanocellular CO2 foaming of PMMA assisted by block copolymer nanostructuration, Chem. Eng. J. 243 (2014) 428-435.

[32] J. Kraus, P. Müller-Buschbaum, T. Kuhlmann, D.W. Schubert, Stamm, Confinement effects on the chain conformation in thin polymer films, Europhys. Lett. 49 (2000) 210-216.

[33] R.G. Kirste, W.A. Kruse, K. Ibel, Determination of the conformation of polymers in the amorphous solid state and in concentrated solution by neutron diffraction, Polymer 16 (1975) 120-124.

[34] G.H. Michler, H.-H.K.-B. von Schmeling, The physics and micro-mechanics of nano-voids and nano-particles in polymer combinations, Polymer 54 (2013) $3131-3144$.

[35] J.L. Keddie, R.A.L. Jones, R.A. Cory, Interface and surface effects on the glasstransition temperature in thin polymer films, Faraday Discuss. 98 (1994) 219-230.

[36] J.L. Keddie, R.A.L. Jones, R.A. Cory, Size-dependent depression of the glass transition temperature in polymer films, Europhys. Lett. 27 (1994) 59-64.

[37] Z. Yang, A. Clough, C.-H. Lam, O.K.C. Tsui, Glass transition dynamics and surface mobility of entangled polystyrene films at equilibrium, Macromolecules 44 (2011) 8294-8300.

[38] J.A. Forrest, K. Dalnoki-Veress, J.R. Dutcher, Interface and chain confinement effects on the glass transition temperature of thin polymer films, Phys. Rev. E 56 (1997) 5705-5716

[39] I. Blaszczyk-Lezak, M. Hernández, C. Mijangos, One dimensional PMMA nanofibers from AAO templates. Evidence of confinement effects by dielectric and raman analysis, Macromolecules 46 (2013) 4995-5002.

[40] J. Teisseire, A. Revaux, M. Foresti, E. Barthel, Confinement and flow dynamics in thin polymer films for nanoimprint lithography, Appl. Phys. Lett. 98 (2011) 013106.

[41] Y. Ding, H.W. Ro, T.A. Germer, J.F. Douglas, B.C. Okerberg, A. Karim, C.L. Soles, Relaxation behavior of polymer structures fabricated by nanoimprint lithography, ACS Nano 1 (2007) 84-92.

[42] T. Ube, H. Aoki, S. Ito, J.-i. Horinaka, T. Takigawa, Conformation of single PMMA chain in uniaxially stretched film studied by scanning near-field optical microscopy, Polymer 48 (2007) 6221-6225.

[43] T. Ikeda-Fukazawa, D. Kita, K. Nagashima, Raman spectroscopic study of CO2sorption process in poly methyl methacrylate, J. Polym. Sci. Part B Polym. Phys. 46 (2008) 831-842.

[44] X. Ma, A.J. Peyton, Y.Y. Zhao, Eddy current measurements of electrical conductivity and magnetic permeability of porous metals, NDT E Int. 39 (2006) $562-568$.

[45] S. Napolitano, S. Capponi, B. Vanroy, Glassy dynamics of soft matter under 1D confinement: how irreversible adsorption affects molecular packing, mobility gradients and orientational polarization in thin films, Eur. Phys. J. E, Soft matter 36 (2013) 61. 